\title{
Políticas universitarias en Argentina: sistema nacional de docentes investigadores universitarios en la mira
}

\author{
University policies in Argentina: national system of university teacher
} investigators in la mira

\section{Políticas universitárias na Argentina: sistema nacional de pesquisadores de professores universitários em la mira}

\author{
Judith Naidorf' \\ Universidad de Buenos Aires, Facultad de Filosofía y Letras, Profesora. \\ https://orcid.org/0000-0001-82155273 \\ Daniela Perrotta ${ }^{2}$ \\ Universidad de Buenos Aires, Faculdad de Ciencias Sociales, Profesora. \\ http://orcid.org/0000-0003-1060-5129 \\ Guido Riccono ${ }^{3}$ \\ Universidad Nacional del Comahue, Profesor. \\ https://orcid.org/0000-0002-5428-5692

\section{Mariángela Napoli ${ }^{4}$} \\ Universidad de Buenos Aires, Profesora. \\ https://orcid.org/0000-0002-4782-4803
}

Resumen: En Mayo de 2019 la Secretaría de Políticas Universitarias del Ministerio de Educación de Argentina crea el Sistema Nacional de Docentes Investigadores Universitarios (SIDIUN) que busca reemplazar el Programa de Incentivos a Docentes Investigadores creado en 1993. En este artículo proponemos analizar las características que asume esta política universitaria en perspectiva histórica. La comprensión del contexto neotecnocrático y privatista que el modelo educativo ha

Doctora en Ciencias de la Educación por la Universidad de Buenos Aires; Licenciada en Ciencias de la Educación por la Universidad de Buenos Aires.

2 Doctora en Ciencias Sociales por la Facultad Latinoamericana de Ciencias Sociales; Magister en Educación por la Facultad Latinoamericana de Ciencias Sociales.

3 Doctor en Ciencias de la Educación por la Universidad de Buenos Aires; Magister en Pedagogías críticas y problemática socioeducativa por la Universidad de Buenos Aires.

4 Licenciada en Letras por la Universidad de Buenos Aires; doctoranda en Ciencias d la Educación en la Universidad de Buenos Aires. 
asumido desde diciembre de 2015 resulta imprescindible para abordar el marco de surgimiento de esta política y los considerandos que la fundamentan. Desde un enfoque descriptivo y analítico basado en investigación documental y de investigación participante recuperamos la teoría generada en el marco del campo de estudios sobre la universidad (KROTSCH; SUASNÁBAR, 2002) a fin de contribuir con un debate que sólo se ha visto reflejado en la crítica periodística y sindical pero con poca presencia en el ámbito de la producción académica. Como conclusión, sostenemos que el gobierno macrista (2015-2019) intentó modificar la reglamentación actual sin una lógica que se proponga perfeccionar las debilidades existentes y en sintonía con las tendencias mundiales en flexibilización y precarización del trabajo docente universitario.

Palabras claves: Sistema Nacional de Docentes Investigadores. Política pública argentina. Trabajo docente universitario.

Abstract: In May 2019, the Secretariat of University Policies of the Ministry of Education of Argentina creates the National System of University Research Teachers (SIDIUN) that seeks to replace the Research Teacher Incentive Program created in 1993. In this article we propose to analyze the characteristics that assume this university policy in historical perspective. The understanding of the neotechnocratic and privatist context that the educational model has assumed since December 2015 is essential to address the framework of emergence of this policy and the considerations that support it. From a descriptive and analytical approach based on documentary research and participant research, we recover the theory generated in the framework of the university field of study (KROTSCH; SUASNÁBAR, 2002) in order to contribute to a debate that has only been reflected in the journalistic and union criticism but with little presence in the field of academic production. In conclusion, we maintain that the Macrista government (2015-2019) tried to modify the current regulations without a logic that aims to improve existing weaknesses and in tune with world trends in flexibility and precariousness of university teaching work.

Keywords: National System of University Research Teachers. Argentine public policy. University teaching work.

Resumo: Em maio de 2019, a Secretaria de Políticas Universitárias do Ministério da Educação da Argentina cria o Sistema Nacional de Professores Universitários de Pesquisa (Sidiun), que busca substituir o Programa de Incentivo ao Professor Pesquisador, criado em 1993. Neste artigo, propomos analisar as características que assuma essa política universitária em perspectiva histórica. A compreensão do contexto neotecnocrático e privatista que o modelo educacional assume desde dezembro de 2015 é essencial para abordar a estrutura de emergência dessa política e as considerações que a sustentam. A partir de uma abordagem descritiva e analítica, baseada em 
pesquisa documental e pesquisa participante, recuperamos a teoria gerada no âmbito do campo de estudos universitário (KROTSCH; SUASNÁBAR, 2002), a fim de contribuir para um debate que só se refletiu na crítica jornalística e sindical, mas com pouca presença no campo da produção acadêmica. Em conclusão, sustentamos que o governo Macrista (2015-2019) tentou modificar os regulamentos atuais sem uma lógica que visasse melhorar as fraquezas existentes e em sintonia com as tendências mundiais de flexibilidade e precariedade do trabalho de ensino universitário.

Palavras-chave: Sistema Nacional de Professores de Pesquisadores Universitários. Política pública argentina. Trabalho docente universitário.

Recebido em 5 de setembro de 2019

Aceito em 14 de abril de 2020

Publicado em 10 de junho de 2020

\section{INTRODUCCIÓN}

En 2019 la Secretaría de Políticas Universitarias del Ministerio de Educación argentino crea el Sistema Nacional de Docentes Investigadores Universitarios (Sidiun) que busca reemplazar el Programa de Incentivos a Docentes Investigadores (Proince) creado en 1993.

En línea con las tendencias mundiales en flexibilización y precarización del trabajo docente universitario se estableció el Proince que permitía el pago diferencial de un incentivo a las tareas de investigación que fueran realizadas por docentes universitarios cuyo salario era despreciable para la época. A cargo de la implementación de esta política estuvo la primera Secretaría de Políticas Universitarias en el marco de un país cuya valoración de la autonomía universitaria era reticente a toda intervención ministerial. El programa de incentivos generó un masivo rechazo de la comunidad académica que tras la ola crítica sucumbió a su modelo, se apropió y olvidó su génesis.

El salario docente universitario en Argentina se encontraba entre los valores más bajos de la historia en el año en que surge la normativa. En valores constantes, el salario de un docente con dedicación exclusiva sufrió una pérdida de 68\% entre 1980 y 1990 (GENTILI, 2001; FERNÁNDEZ LEMOS; WINAR, 1997). Al igual que en 2019 la norma surge en un contexto de restricción y pérdida del poder adquisitivo que promedió el $30 \%$ del salario del docente universitario y una merma en los fondos destinados a la investigación. En este contexto similar la resistencia se manifiesta nuevamente menguada por la necesidad de los profesores de aceptar una propuesta que suponga algún tipo de incremento salarial. 
A fin de comprender el rol de las universidades privadas argentinas $y$ antes de continuar con la caracterización de los antecedentes que desembocan en el Sidiun (FEDERACIÓN NACIONAL DE DOCENTES UNIVERSITARIOS, 2019) intercalaremos un apartado que permite contextualizar la forma que éstas adquieren en Argentina a través de una análisis de fuentes históricas y documentales; también se realizará el estudio de datos a partir del análisis del marco teórico basado en el campo de investigación de la universidad latinoamericana y el rol del estado en el devenir de un modelo neotecnocrático.

\section{BREUE RECORRIDO POR EL MECANISMO DE CATEGORIZACIÓN Y DEL INCENTIUO DOCENTE}

En trabajos anteriores, afirmábamos que el Proince se había convertido en uno de los principales instrumentos de la reforma y el reajuste (NAIDORF, 2005). El mismo consiste, desde su primera implementación en 1993, en un plus salarial que se paga en tres cuotas anuales fijadas según una categorización que se establece en base a indicadores de productividad. La categorización es el proceso de evaluación de los antecedentes de un docente de una universidad nacional por pares evaluadores, para asignarle una jerarquía. En un principio, se fijaron cuatro categorías (de la $A$ a la $D$ ) en las que los docentes eran clasificados según el desarrollo de sus tareas de docencia e investigación. En 1999 se produjo una recategorización que en una escala de I a V de Docente-Investigador. Asimismo esta categorización resultó errante, asincrónica, asociada a los vaivenes de la política nacional y sin periodicidad previsible.

El proceso de categorización se ha abierto cada 4 años, aproximadamente, y sólo podían postularse los docentes rentados len Argentina existe una masa significativa de docentes sin renta o ad honorem); asimismo, no está asociado de manera directa al incentivo salarial. Este sistema de categorización y evaluación impone un sistema paralelo al de los concursos docentes y es optativo. Para no quedar excluido de este programa, todo investigador debe acreditar tareas de docencia y todo docente debe investigar (VOMMARO, 2001). Este sistema de incentivos, entonces, genera una gran estratificación dentro del sector docente y significó una deshomologación salarial al exponer esta doble caracterización (y luego exigencia) de las tareas de docencia e investigación que no estaban contempladas (LEAL, 2002).

Los fondos de este programa son manejados en forma centralizada por el Ministerio de Educación a través de la Secretaría de Políticas Universitarias (SPU) y las cuotas son abonadas con atrasos significativos que pueden sobrepasar el año (NAIDORF, 
2005). En la práctica, las categorías establecidas en principio como A, B, C y D cuyo impacto era relativamente significativo fue mermando $y$ discontinuándose las convocatorias $y$ concomitantemente retrasándose en años el pago de las cuotas correspondientes al mismo. A diferencia de modelos contemporáneos en el momento de su creación como el Sistema Nacional de Investigadores en México, este programa se ha caracterizado por el retraso considerable en el pago de las cuotas y en la poca significatividad de los montos que se asignan. El mismo corresponde a un pago no remunerativo y no bonificable. Así, a diferencia del sistema mexicano cuya convocatoria anual implica un porcentaje muy alto del honorario docente universitario para quien lleva a cabo tareas de investigación

En el caso argentino el proceso de categorización docente fue abierto sólo en las siguientes ediciones: 1993, 1996, 1998, 2004, 2009, 2011 y 2014. En octubre de 2011, el Programa de Incentivos convocó un llamado especial para categorizar para aquellos docentes-investigadores que: a) hubieran retornado al país y al ámbito universitario a través de programas oficiales de reinserción que, b) no tuvieran una categoría vigente, c) nunca hubieran obtenido una categoría d) no se hubieran presentado en 2009 por no cumplir con la exigencia relativa al cargo docente mínimo necesario para obtener una categoría superior, requisito exceptuado posteriormente. Este último proceso de categorización obtuvo una amplia participación, que alcanzó a 5.539 postulantes, superando las expectativas de la Coordinación del Programa y mostrando la creciente demanda de los docentes e investigadores a un reacomodamiento y actualización.

El rol de la SPU, resistido en los tiempos de su creación, fue adquiriendo legitimidad así como la Comisión Nacional de Evaluación y Acreditación Universitaria (Coneu) por su rol de dique de contención de las iniciativas privadas de baja calidad que solicitaban su acreditación. Los evaluadores y acreditadores se conformaron como pares evaluadores que, en un marco de libertad de discusión de los casos, se pronuncian en dictámenes que son acatados por el organismo. La misma SPU dependiente del Ministerio de Educación de la Nación creó planes de fomento que acompañaron a las universidades en sus proyectos de mejora de mecanismos de extensión, movilidad, internacionalización, entre otras.

Actualmente, el pago de incentivos posee un retraso promedio de dos años en el cobro y los montos son muy bajos, como se puede ver en la siguiente Tabla: 
Tabla 1 - Proporción monto incentivo/salario docente universitario bruto con antigüedad promedio, 2004

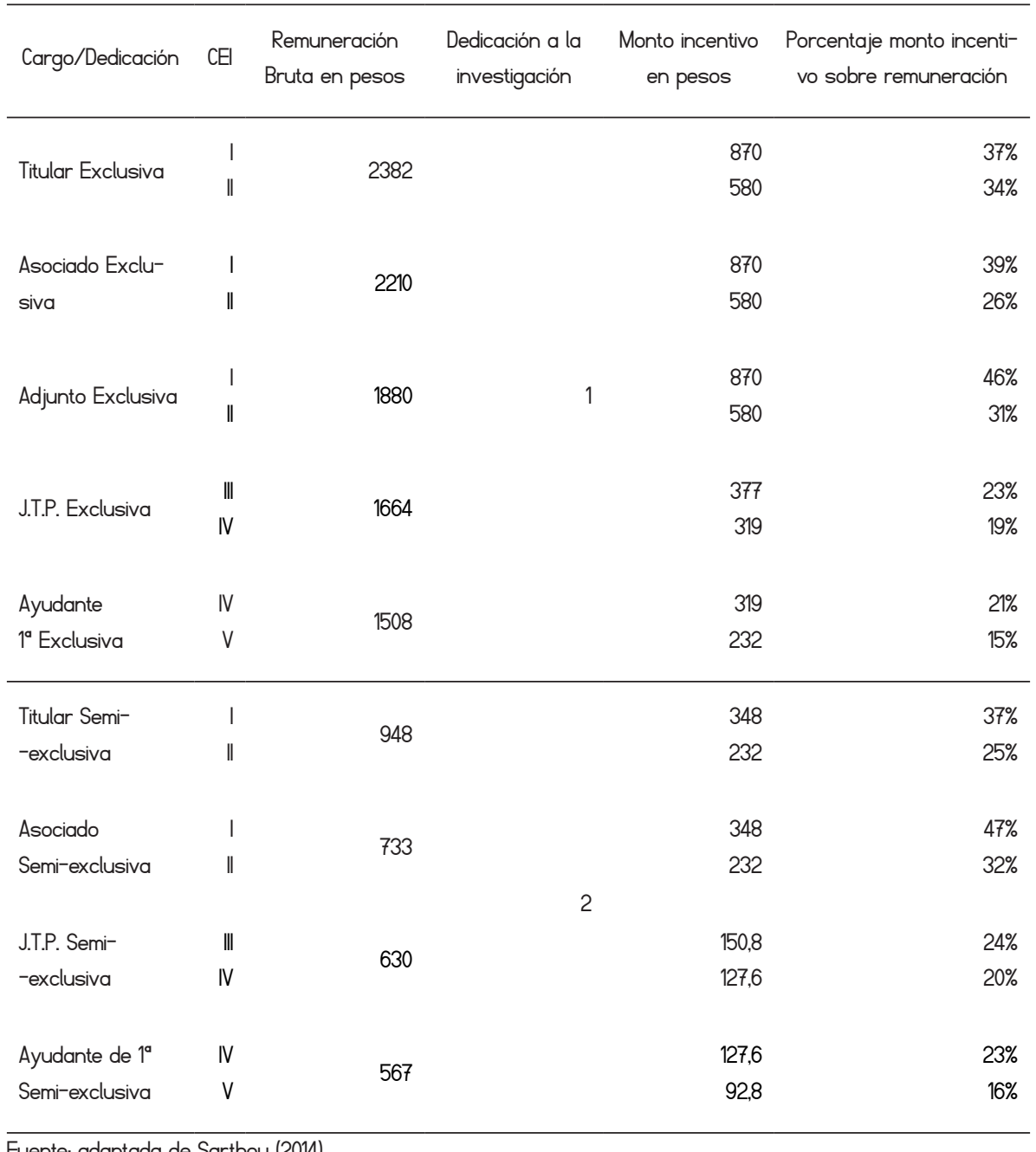

Fuente: adaptada de Sarthou (2014).

El programa siempre fue voluntario y cuenta en la actualidad con unos 25.000 profesores categorizados de una planta de 130.000 docentes universitarios en universidades nacionales de los cuales el solo el $11 \%$ corresponde a cargos de dedicación exclusiva y $11 \%$ a dedicación semiexclusiva. Se reconocen casos de resistencia colectiva que fueron limitados en el tiempo así como manifestaciones individuales que no llegaron a resultar resonantes - significativas. Los beneficiarios del programa eran los docentes e investigadores de universidades públicas (en la que están enrolados el $80 \%$ de los estudiantes de Argentina). 
Asimismo cabe diferenciar las características que ha asumido la categorización por un lado y el cobro de incentivos por el otro. La categorización fue adoptada por casi todas las universidades y organismos de ciencia y tecnología como parámetro común de diferenciación del personal docente. Así por ejemplo algunas universidades establecen como requisito para dirigir proyectos de investigación estar categorizado y en algunos casos específicamente es requerida la categoría 1, 2 ○ 3 para aspirar a ejercer funciones dentro del ámbito investigativo. Para el sistema científico tecnológico y como medida equivalente del escalafón de investigador científico se considera que un profesor universitario que reviste la categoría 1 y 2 corresponde al equivalente a investigador formado o independiente.

Por su parte, el incentivo asociado a la categoría sólo es percibido por los docentes con dedicación exclusiva o investigadores y becarios de Consejo Nacional de Investigaciones Científicas y Técnicas (CONICET) que lleven a cabo tareas docentes en la universidad en complementariedad con su tarea investigativa. El Conicet es un organismo descentralizado en el ámbito del Ministerio de Ciencia, Tecnología e Innovación Productiva de la Nación; este organismo, a través de la Carrera del Investigador Científico (CIC), así como mediante el otorgamiento de becas de formación en la investigación, es el principal "empleador" de científicos en la Argentina, junto con las universidades, que emplean a docentes que también realizan investigación científica. Estos dos canales o vías de profesionalización de docentes-investigadores continúan problematizando las elecciones de miles de jóvenes, a la hora de pensarse dentro del sistema universitariocientífico argentino.

Por ende, esta política de categorización e incentivos ha sido errática, poco articuladas entre sí y con el resto de la sociedad tal como corresponde al complejo de educación superior que expone lo anteriormente explicitado (NAIDORF; PERROTTA, 2010, p. 197-198). Esta característica que asumía el complejo de educación superior había comenzado a articularse a partir de la creación del Ministerio de Ciencia, Tecnología e Innovación Productiva en 2007 y reducido a Secretaría en 2018; sin embargo, aún persiste la coexistencia de modelos cuasi paralelos con problemas de vinculación entre trayectorias de investigadores y docentes.

La recuperación de su salario durante el período 2005 y 2014 fue más evidente en el caso de los salarios con mayor dedicación y los dedicados a la investigación. Esta diferencia de los ingresos entre docencia e investigación ha generado una lectura negativa de la docencia en desmedro del dictado de clases como actividad central ya que no se promovería la investigación de calidad en las universidades, sino la competencia en la búsqueda individual de resultados acreditables para una evaluación favorable del 
desempeño (RINESI, 2012). Ante la presencia de dos circuitos diferenciados (investigación/ docencia universitaria) se comprende cómo el Sidiun pretende simplificar en una supuesta carrera los problemas que derivan de estos perfiles que aún siguen sin resolverse en el sistema universitario argentino. Asimismo, la importancia de esta diferenciación a partir de la identificación del Sidiun con una nueva forma de categorización docente no asociada necesariamente a los incentivos económicos que se suponen concomitantes.

Sumado a esto, durante el transcurso del tiempo de consolidación del programa, éste fue adquiriendo matices no considerados en primera instancia. Las universidades y los organismos de promoción de la ciencia y la tecnología comenzaron a utilizar la categorización como unidad de medida para los concursos docentes en las universidades, la habilitación para la dirección de proyectos, la posibilidad de dirigir becarios, y como condición del plantel docente en instancias de evaluación institucional y de acreditación de carreras de grado y posgrado, principalmente.

En el año 2014, previo al fin del gobierno de Cristina Fernández de Kirchner se llamó a la categorización opcional para quienes habían sido categorizados en 2009 y obligatoria para quienes hayan obtenido la categoría con anterioridad. Tal como ocurrió desde los inicios del programa en 1993 sólo los docentes - investigadores de universidades públicas podían acceder a dicho beneficio. En junio de 2015 se creó el Programa de categorización de docentes investigadores universitarios que tuvo por objeto categorizar a los docentes investigadores de las universidades de gestión privada; de las instituciones universitarias dependientes de las Fuerzas Armadas dependientes del Ministerio de Defensa (MinDef) y de las instituciones universitarias de las Fuerzas Policiales y de Seguridad dependientes del Ministerio de Seguridad (MinSEg), aplicando para ello los criterios del Proince (Programa de incentivos a los docentes investigadores de universidades nacionales).

Las actividades que implicaron el proceso de categorización de los investigadores se inició con significativo retraso dos años después de su convocatoria. En 2020 aún no habían sido comunicadas la totalidad de las resoluciones sobre categorizaciones. Esta imposibilidad del sistema de dar cuenta de los cambios en la actualización y acumulación de antecedentes de los y las docentes investigadores fue utilizada como argumento a favor del Sidiun que prometía una agilización de los procesos a través de nuevos considerandos. El Sidiun es una avanzada hacia una pretendida armonización de los denominados subsistemas público y privado a partir de una categorización única para docentes e investigadores, consecuencia central de este proyecto que se suma a los ya mencionados. 


\section{LAS UNIUERSIDADES PRIUADAS EN ARGENTINA}

Podemos remontarnos a los años '30 del siglo XX cuando Eduardo Braun Menéndez $(1945,1957)$ y el propio Bernardo Houssay soñaban con una universidad privada de investigación al estilo Harvard. Sus motivaciones provenían del campo de la "libertad" académica que suponían al espacio privado en contra de la regulación y politización estatal de la investigación científica y de las interpretaciones que de la autonomía universitaria han tenido lugar en Argentina. Cabe aclarar que la autonomía como endogamia (NAIDORF, 2009) se manifiesta frente a la supuesta "libertad" académica que promovió y se manifestó en la práctica en la adopción de una agenda de investigación cuyos temas eran definidos estratégicamente, a partir de una valoración social del producto del conocimiento- en otras latitudes, principalmente en los países centrales.

Más adelante, el recordado episodio que dividió en las calles a la sociedad argentina denominado "Laica o Libre" tuvo lugar en 1958 y enfrentó a dos modelos antagónicos de la educación superior en Argentina, encabezados por los hermanos Frondizi - Arturo como presidente y Risieri como rector de la Universidad de Buenos Aires (UBA).

Laica era el modelo de universidad pública que poseía larga data en el país cuya primera universidad se funda en 1613 nacionalizada en 1820 y fue escenario de la Reforma del १८'. La Ley Avellaneda (1885) establecía que las universidades nacionales eran las podían expedir títulos oficiales. Los sectores eclesiásticos fueron quienes iniciaron la demanda de una educación universitaria que llamaron Libre, es decir, que pueda equipararse a la educación pública en tanto su capacidad de expedición de títulos y que le permitiera hacerlo acorde a sus creencias. Disimulado bajo el significante Libre, este objetivo se consiguió mediante la Ley Domingorena del año 1958, luego del acto de protesta que encabezó el entonces rector José Luis Romero de la UBA quien renunció a su cargo en un intento previo de promulgación de la misma en el año 1956. Esta disputa tuvo como protagonistas a los hermanos Fronidizi (Risieri rector de la UBA en defensa de la educación Laica y Arturo, presidente de la nación quien apoyaba la universidad libre). Las primeras universidades privadas fueron confesionales y recién en los años ochenta y noventa en consonancia con las tendencias internacionales de mercantilización de la universidad fueron creadas universidades privadas bajo la forma de fundaciones dado que aún no existen en Argentina universidades -tal como se reconoce en Brasil- con manifiesto ánimo de lucro al menos en su autodefinición. 
Sin embargo, hasta hoy en día, la matrícula del estudiantado universitario es mayoritariamente pública acompañada de una constante apertura de nuevas universidades públicas por etapas (Proyecto Taquini en los años '70, nuevas universidades en los años '90 y en los 2000) como apuesta a un Estado presente en el conurbano de la provincia de Buenos Aires donde la oferta de educación superior era escasa y la existente se ubicaba en manos de entidades privadas. Producto de las presiones ejercidas por los organismos de acreditación que les otorgan validez a los programas de grado y posgrado movidas por el interés por desarrollar un negocio rentable - muy tardío en Argentina comparado con México, Brasil, Colombia - y, en algunos casos, por el interés de desarrollar líneas de investigación cuyos temas permiten el acceso a financiamientos internacionales, las universidades privadas comenzaron a abrir laboratorios de investigación. Asimismo la oportunidad de poseer Argentina una masa crítica de nuevos doctores sin espacio laboral en las universidades públicas sirvió de apoyo para este fenómeno.

El Conicet así como la Agencia Nacional de Promoción de la Ciencia y Tecnología (ANPCyT) no discriminaron entre universidades públicas y privadas. Además, la ampliación de la inversión en ciencia y tecnología que se inició en 2005 y llegó hasta 2015 benefició a las universidades privadas con becas y subsidios. La cantidad de investigadores del Conicet en universidades públicas y privadas se triplicó entre el 2004 y el 2012, elevándose de 71 a 179; y aún más impactante fue el crecimiento en la cantidad de becarios de doctorado y postdoctorado, aumentando más de seis veces en dicho periodo (de 23 a 143 becarios) (ADROGUÉ et al., 2014).

De igual manera, con sus propios esquemas de ingreso, permanencia y ascenso en la docencia universitaria -sin concursos públicos (como es el caso de las Universidades Nacionales) sin sindicalización ni formando parte de negociaciones paritarias-, estas instituciones pueden competir por ciertos fondos en igualdad de condiciones que las universidades públicas. Ya la Ley de Educación Superior (LES) promulgada en 1995 refería al sistema de educación superior pública y privada (denominándolos como subsistemas), en línea con la definición plasmada en 1993 en la Ley Federal de Educación que hacía referencia a la educación de gestión pública y privada (ARGENTINA, 1995). Nacidas bajo el seno del modelo neoliberal, las dos leyes poseían el objetivo de hacer porosa la frontera entre lo público y lo privado en una igualación de sus funciones con y respecto a la sociedad. Esta igualación o armonización en el lenguaje, generó un aumento de la repartición de los fondos públicos que las instituciones privadas reciben, así como la LES y la LFE promovieron la mercantilización de la educación.

En efecto, el artículo 66 de la LES promueve la común recepción de subsidios del Estado y exime a universidades privadas del pago de impuestos y contribuciones nacionales (FERNÁNDEZ; RUIZ, 2002). Esto permite asociar al nuevo proyecto del Sidiun con 
el problema de cierto financiamiento de universidades privadas con fondos públicos sin ningún tipo de retribución por parte de estas últimas; se visualiza que esto ya se había gestado en los años '90 en Argentina y se retoma, bajo un modelo neotecnocrático, en la actualidad.

A diferencia de lo que ocurre en Brasil, en algunas universidades denominadas particulares $o$ en otras latitudes, las universidades privadas en Argentina no se caracterizan por un salario significativamente mayor (salvo en algunos posgrados) sino que ofrecen comodidades a sus docentes en términos edilicios, administrativos y burocráticos. Además resultan un espacio de trabajo para quienes no logran obtener una plaza en una universidad pública.

Los montos destinados para investigación que históricamente han llevado a cabo las universidades privadas corresponden a un $10 \%$ del valor que invierten las universidades públicas. Globalmente, para el año 2011, las universidades privadas declararon la presencia de 3.237 investigadores de los cuales 193 pertenecían a carreras en el Conicet y 138 eran becarios en esta institución, lo que expande normas de investigación y representa el financiamiento de origen estatal más relevante para las universidades privadas, llegando a un $20 \%$ del gasto total de estas instituciones en este rubro (BARSKY; CORENGIA, 2017).

Este panorama permite explicar por qué consideramos que éstas aún no se encuentran en un estado de desarrollo tal que permita igualdad de condiciones, disfrazada en la norma con la alusión a la "armonización", aspecto en el que nos detendremos más adelante. Si bien aumentó la investigación orientada en institutos universitarios focalizados en el área de la salud, la mayor cantidad de instituciones del sistema no reflejó cambios importantes en la actividad de investigación en respuesta a estas políticas públicas de inversión en ciencia y tecnología mencionadas (ADROGUÉ et al., 2014). Esta paridad está reflejada en la normativa que regula al Sidiun dada la participación previsible en los órganos de control y gestión del programa así como en el acceso a categorías. Para ser más específicos, tal como estaría previsto, los docentes sin concurso de las universidades privadas podrían ser evaluadores de docentes concursados de universidades públicas. Esto permite sospechar que se estarían equiparando a los docentes investigadores de instituciones públicas con los del sector privado, sin alertar que los objetivos, finalidades y sentidos de ambos sistemas son completamente diferentes. 


\section{EUALUACIÓN DEL DESEMPEÑO, PROMOCIONES Y CRITERIOS DE UALIDACIÓN DEL SIDIUN}

El sistema de evaluación argentino se basa principalmente en el desempeño de tareas en general "ad honorem" o con una paga simbólica que cumplen las y los investigadores de categoría I y II del sistema nacional de incentivos. En el caso del Conicet, los bancos de evaluadores con que cuentan no superan las mil personas y que dado lo voluntario de la aceptación de la función se encuentra saturado ese grupo de potenciales evaluadores.

El proceso de categorización 2014 hubiera permitido ampliar dicha base si el proceso se hubiera terminado. Las dificultades en el retraso en el arribo a resultado y en el tardío proceso de inicio de los encuentros evaluativos (en 2016) tuvieron principalmente que ver con el retraso en la adjudicación de sedes, los problemas que las mismas tuvieron para el recupero de fondos (debiendo adelantar y asumir ellas gastos que corresponden al Ministerio de Educación) y otros problemas evitables que jugaron un papel clave en el descontento al que aluden para elevar esta nueva propuesta.

Estudios como el llevado a cabo por el equipo que coordina Fernanda Beigel (2009) desde la Universidad Nacional de Cuyo en Mendoza permitieron comprender algunas de las características observadas a lo largo del proceso de adjudicación de categorías tal como son las siguientes:

\footnotetext{
El Proince jugó un papel central en la subsistencia de perfiles diversos de producción y circulación del conocimiento, en el desarrollo de las revistas cientificas argentinas y en la distribución del presupuesto de investigación destinado a las universidades nacionales. El estudio también permitió observar una serie de debilidades y tensiones: la grilla de evaluación estimula un tipo de evaluación más burocrático-administrativa que cognitiva, con lo cual pierde incidencia en la consolidación de la investigación que se desarrolla en las universidades; existen desigualdades regionales e institucionales estructurales que se reflejan en la distribución de los docentes incentivados; el proceso de categorización es excesivamente largo y las convocatorias se extienden entre períodos de cuatro años o más; el sistema de evaluación es conservador en el sentido de que tiende a consolidar las jerarquías existentes entre los docentes-investigadores. (BEIGEL; BEKERMAN, 2009, p. 30).
}

Con anterioridad Ana de Fanelli y Marina Moguillansy (2010) habían reconocido que el programa ha desembocado en una ampliación de la base de docentes universitarios que se autopercibían como investigadores, se ha incentivado el cumplimiento de mayores participaciones en actividades de investigación por parte de los docentes junto con el crecimiento de los posgrados, la ampliación de las vacantes para becas y en la carrera 
de investigador científico- tecnológico entre 2006 y 2015. El incremento de docentes que se percibían como investigadores entre 1994 y 2006 había sido de un 77\% según las autoras.

La diversificación y ampliación de la planta de docentes e investigadores llevaron a un camino en plena revisión de indicadores de productividad medido principalmente en producción de papers, títulos de posgrado, participación y dirección de proyectos de investigación, formación de tesistas y becarios, etc. que rige, sobre todo, en el Conicet (NAIDORF; PERROTTA, 2017). Sin embargo, la riqueza de los procesos evaluativos sigue gravitando en la discusión sobre el proyecto y la valoración global y particular de cada caso por parte de pares. En consecuencia, el Sidiun se apoya en las cuestiones más debatidas por diversos autores y que acentúan los perfiles de docentes-investigadores ya señalados.

El Sidiun (Resolución del Ministerio de Educación N 1216/19), se presenta como una supuesta economía de esfuerzos, pretende reducir al mínimo estas instancias al centralizar la categorización en un grupo reducido con equidad y paridad entre docentes investigadores de universidades públicas y privadas y representantes del Consejo Interuniversitario Nacional (CIN, órgano de coordinación de las universidades nacionales) y Consejo de Rectores de Universidades Privadas (CRUP, órgano de articulación de instituciones privadas). El sistema en consonancia con las normativas y decretos impuestos por la gestión actual- fue lanzado sin discusión ni debate previo con las organizaciones que representan colectivamente a la docencia universitaria, ni las instancias de articulación de las instituciones lo cual tendrá un alto impacto en la orientación y desarrollo del sistema universitario. En resumen, esta situación ha llevado a las centrales gremiales de docentes universitarios a expresar denuncias en torno a la equiparación de las universidades públicas y las universidades privadas entre otros considerandos en torno al Sidiun.

Por último, y en consonancia con el concepto de autonomía también mencionado y como un valor fundante de las universidades argentinas, se teme que el Sidiun afecte la plena vigencia del Convenio Colectivo de Trabajo (CCT) de las Universidades Nacionales. El CCT establece que cada vez que un docente sea evaluado los gremios tienen que designar veedores para garantizar la transparencia y objetividad de la evaluación. En el nuevo sistema se profundiza la orientación de la evaluación centralizada de la docencia en sus actividades de investigación por el sistema nacional, relegando así el propio papel de cada universidad y de sus unidades representativas. 


\subsection{CATEGORIZACIÓN NO IMPLICA INCENTIUOS}

Tal como afirmábamos anteriormente el Sidiun no implica incentivo docente alguno, al menos tal como fue presentado a lo largo y ancho del país tanto en universidades públicas como en privadas de manera indistinta por parte del Ministerio de Educación de la Nación. Un manifiesto desencanto producto de los problemas asociados a la implementación del Programa de Incentivos y el contexto de descrédito a las universidad y la ciencia por parte del gobierno de Mauricio Macri impidieron un movimiento de resistencia más evidente más allá de las contundentes declaraciones en contra por parte de las centrales sindicales de docentes universitarios como Conadu (Federación nacional de docentes universitarios), Conadu-H (Federación nacional de docentes investigadores y creadores universitarios), Agd (Agrupación gremial docente) y Fedun (Federación de docentes de universidades). Las condiciones laborales y la baja en los salarios que representa una caída del poder adquisitivo desde 2015 podría ser una explicación para una actitud aún pasiva del profesorado argentino frente a esta propuesta.

Las preguntas de los asistentes a las reuniones explicativas (algunas de ellas llevadas a cabo en universidades privadas) han estado ligadas principalmente a saber cómo ajustarse a la norma y a los incentivos y se ha aclarado que esto se trata de un cambio en la categorización, no una promesa de incentivos. Por ende, no asociada a un compromiso con un pago adicional al salario docente el Sidiun exagera en las pretensiones que enuncia en los considerandos que pueden consultarse en la primera página del Reglamento del Sistema Nacional de docentes investigadores, Sección I, Artículo $1{ }^{\circ}$.

Se trata, afirman, de un reconocimiento o jerarquización aunque principalmente apelan a la armonización. Detengámonos en la etimología de dicha palabra. Armonización significa según la real academia española "Poner en armonía, o hacer que no discuerden o se rechacen dos o más partes de un todo, o dos o más cosas que deben concurrir al mismo fin" (https://www.rae.es/drae2001/armonizar). Se armoniza lo equivalente o lo que se pretende que así sea, pero siempre a partir de alguna base común. En este caso se eleva aún la apuesta iniciada en 1956 de otorgar igual valoración a la universidad pública y a la privada en un contexto donde, más allá de las contribuciones de la Coneau a regular el negocio de la educación superior, el balance sigue siendo muy desigual en términos de contribución, por ejemplo, al desarrollo nacional. 
La creación de nuevas universidades públicas en las más diversas latitudes del territorio argentino y la creación de más espacios de formación a distancia hace desestimar el argumento acerca del cumplimiento de un supuesto rol social que cumplirían las universidades privadas en la ampliación y pluralismo de corrientes de pensamiento que cumplen estas ofertas educativas. La afirmación de que se orientan a una oferta más exclusiva o elitista por la que los clientes-alumnos puedan querer optar no es motivo suficiente para hacer uso de los aportes estatales de reconocimiento mediante categorizaciones - siendo que en 2015 ya se había creado un programa exclusivo para universidades privadas- así como tampoco el financiamiento de becas, subsidios y otras exoneraciones fiscales.

Sobre la ampliación de una variedad en la oferta académica tampoco se constituye en un argumento por el cual el Estado deba implicar a las universidades públicas al imponer indirectamente que los solicitantes de las categorizaciones sean docentes cuyos requerimientos académicos son dispares respecto de los propios. Asimismo, un posible argumento en la mira sobre el nuevo sistema de categorizaciones puede residir en la puesta en cuestión de un modelo de universidad que pretende asemejarse al del actual Conicet. El sistema de evaluación de la performance por papers vigente asociado a la noción de productividad puesto en revisión en los últimos años, evidencia que esta pretensión de homologación es también un riesgo que puede acrecentar el Sidiun y que vendría a modificar los supuestos de la cultura académica. Este argumento se enlaza con la idea de que el Sidiun se propone mejorar la eficiencia de gestión del anterior Proince divide aún más los perfiles universidades nacionales Conicet ya señalados.

El Sidiun reforzaría así la devaluación de la docencia como función colectiva, al establecer una categorización sobre la base de la evaluación de las trayectorias individuales de las y los docentes que puedan demostrar la realización de actividades de I+D y en consideración del nivel acreditado en la formación de posgrado. Al mismo tiempo, la formulación del Programa revela una concepción reductiva de la producción de conocimiento en las universidades, limitando sus resultados acreditables a aquellos que pueden inscribirse en la perspectiva específica de la $+\mathrm{D}$, entre los que difícilmente puedan inscribirse los desarrollos de las artes o de las humanidades o en relación con proyectos extensión o que impliquen alguna "misión social".

La actual categorización de docentes universitarios ha promovido una valoración positiva en la formación de recursos humanos (otorga casi 200 puntos), elemento que no contaba en la anterior categorización. Esta situación permitió que muchos docentes-investigadores se volcaran a la tarea formativa de investigadores y becarios. Sin embargo, se visualiza un caótico intento de homogenización, no estando 
claras las definiciones específicas ni la ponderación de los métodos de categorización propuestos. Por todo esto, el argumento sobre la mayor eficiencia que presupone el Sidiun respecto de la anterior categorización cae por su propio peso: con financiamiento y buena gestión el Proince no necesariamente resultaba ineficaz más allá de los condicionamientos asociados detallados anteriormente.

Los sistemas son perfectibles y es función de la SPU hacer los ajustes necesarios. Sin embargo, en esta propuesta se demuestra la falacia del argumento de la eficiencia por el simple hecho de comprender que corresponde a una lógica que ha primado en el gobierno de Macri: la apelación a las políticas públicas que igualan, cuando no argumentan, que lo privado es mejor que lo público en tanto vieja receta neoliberal.

Por otro lado, destacamos que la mención a la periodicidad en la convocatoria está solo anunciada pero no normada. El Proince no preveía tener retrasos significativos en la periodicidad de las convocatorias ni tampoco que los montos asignados sufrieran una desactualización en relación a la inflación y otros factores así tampoco que los mismos fueran percibidos por los investigadores con hasta dos años de retraso. Dado que el problema no era intrínseco de la normativa tampoco lo es el reemplazo de la misma por otra en nombre de la solución a dichas deficiencias de gestión. En el caso del Sidiun se enuncia que cada dos años se prevé la convocatoria a categorizaciónEsto permitiría agilizar la actualización de antecedentes y permitir la promoción entre categorías. Sin embargo en la normativa esto no se garantiza esta periodicidad y nuevamente cabe reiterar que no es problema del Proince la justificación de los retrasos y la falta de periodicidad de las convocatorias.

Por ende, sumado a la incertidumbre de asociar a la nueva normativa con una supuesta eficiencia del sistema se visualiza un corrimiento del debate sobre la cuestión presupuestaria como problema de fondo. Como ya fue mencionado, se sigue evitando la problemática histórica de los escasos y tardíos fondos para la docencia y se pretende redirecccionar el eje hacia una supuesta eficiencia de formas y jerarquías que no acredita los pagos atrasados y solo contribuye a la conformación un estado neotecnocrático que, desde su llegada en 2015, nada puede resolver en el campo de lo práctico y lo palpable. 


\section{UNA NORMATIUA SIN PERSPECTIUA DE GÉNERO NI DE AMPLIACIÓN DE DERECHOS}

El Sidiun supone la posibilidad de solicitar la suspensión de la actividad investigativa por motivos de la asunción de un cargo de gestión u otro motivo. Dado que la categorización es finita, la extensión de la categoría puede ser un beneficio en virtud de la afectación del rendimiento medido con los parámetros en ciencia y tecnología,

Para la consulta sobre las licencias por maternidad o la medición de este rendimiento durante el período de crianza de hijos la respuesta fue que deberían solicitar la suspensión de la actividad académica en esos casos, a fin de solicitar la extensión de la categoría obtenida. Por ende, en el caso de la categorización no se contemplan en la resolución los casos de embarazo o puerperio si al convocarse la misma, se superpone con algunos de esos periodos atravesados por las investigadoras. Esta normativa se encontraría retrasada respecto de los derechos obtenidos por las investigadoras y docentes universitarias,

Asimismo, tampoco se mencionan la valoración del trabajo docente que implique una producción del conocimiento en relación con sectores extra académicos, un reclamo que también cobró vuelo y se ha puesto en discusión en los últimos años. Al establecer una categorización sobre la base de la evaluación de las trayectorias individuales de docentes que puedan demostrar la realización de actividades de investigación, se suprime la condición en la participación en equipos de trabajo que sí se preveía como condición en el anterior programa, lo que fomentaría aún mas el perfil individualista en las trayectorias.

En base a criterios - una vez más - productivistas, el Sidiun tampoco hace referencia al trabajo docente en carrera de grado y eso habilitaría a docentes que sólo trabajen en formación de posgrado puedan llegar a categorizarse, como resultado, se podría desestimular el trabajo docente en las carreras de grado.

Por último, debemos mencionar que el Proince se rige por un sistema regional para llevar adelante el proceso evaluativo y posterior categorización. Esto pone en evidencia, por un lado, que el proceso de discusión se desarrolla en cada una de las regiones (noroeste, noreste, sur, etc) atendiendo las particularidades y las desigualdades del propio sistema en niveles de formación y acceso al conocimiento que se derivan de éste. Con el nuevo Sidiun se plantea el mecanismo opuesto: la centralización; se presuponen evaluaciones en Buenos Aires, eliminando -una vez más- las instancias de discusión, es decir, solo se se prevé la suma de indicadores para conformar las 
categorías. No solo no se resuelven los conflictos mencionados, sino que se agravan con la supresión de instancias de debate regionales.

\section{CONCLUSIONES PRELIMINARES}

A través de una revisión histórica y contextuada del surgimiento del Proince sostenemos que es a través de la negociación paritaria y la revisión de los perfiles docentes investigadores orientados al desarrollo social, a la producción de conocimientos para el bien común y el trabajo colaborativo y solidario que deberían establecerse los parámetros para definir el salario docente y las categorías dentro del sistema.

En distintas oportunidades nos hemos pronunciado críticamente frente a los incentivos o pagos por productividad y otras medidas que impiden comprobar que existan indicadores simples y medibles que permitan ponderar la función del científico la científica y del intelectual asociada a la docencia universitaria. Sin embargo, dado que supone una medida que impactará en la actividad académica es que procuramos contribuir con el análisis de la misma que podrían ponerse en funcionamiento. La ausencia de consulta y participación de instancias representativas de la docencia universitaria en la elaboración del programa se condice con el modus operandi que ha tenido lugar entre 2015 y 2019.

Asimismo, en tiempos de crítica generalizada al futuro de la universidad a principio de los años 2000 habíamos apelado a la comprensión de uso de la palabra "crisis" como preludio de atacar, reformar y refundar una respuesta a la misma. Entender la "crisis" como resistencia, al decir de Boaventura de Sousa Santos (2007, p. 15), supone involucrar la promoción de alternativas de investigación, de formación, de extensión y de organización y recuperar capacidad de definir la crisis contra-hegemónicamente, es decir con autonomía, de modo que la sociedad se vea reflejada en ella. Por ello es que nuestro análisis se propone difundir y criticar los alcances de esta medida que, hasta hoy, no ha sido puesta en funcionamiento. 


\section{REFERENCIAS}

ADROGUÉ, C. et al. La investigación en las universidades privadas de la Argentina. Cambios tras las políticas de aseguramiento de la calidad y financiamiento competitivo. Revista lberoamericana de Calidad, Eficacia y Cambio en Educación, Madrid: Universidad Autónoma de Madrid, v. 12, n. 3 , p. 73-91, 2014.

ARGENTINA. Ley n. 24.521, de 20 de julio de 1995. Ley de educación superior. Boletín oficial, Buenos Aires, 20 jul. 1995. Disponible en: http://servicios.infoleg.gob.ar/infoleglnternet/anexos/25000-29999/25394/texact.htm. Acceso en: 7 abr. 2020.

ARGENTINA. Reglamento del sistema de docentes investigadores, de 7 de mayo de 2019. Ministerio de Educación, cultura, ciencia y tecnología. Boletín Oficial, Buenos Aires, 7 mayo 2019. Disponible en: https://www.argentina.gob.ar/normativa/nacional/resoluci\%C3\%B3n-1216-2019-322832/ texto. Acceso en: 15 abr. 2020.

BARSKY, 0.; CORENGIA, A. La educación universitaria privada en argentina, Revista Debate Universitario, Buenos Aires: Universidad Abierta Interamericana, v. 5, n. 10, p. 31-70, mayo 2017.

BEIGEL, F. Autonomía y Dependencia académica: universidad e investigación científica en Chile y Argentina (1950-1980). 1. ed. Buenos Aires: Biblos, 2010.

BEIGEL, F.; BEKERMAN, F. Ciencia, productividad y desigualdades. Diario Página 12, Buenos Aires, 3 jul. 2009. Disponible en: https://www.pagina12.com.ar/204167-ciencia-productividad-y-desigualdades. Acceso en: 7 abr. 2020.

BRAUN MENÉNDEZ, E. Las etapas para la creación de una universidad privada. Revista Ciencia e Investigación, Buenos Aires: Asociación Argentina para el Progreso de las Ciencias Comité Editorial, v. XIII, n. 3, p. 98, 1957.

BRAUN MENÉNDEZ, E. Universidades no oficiales e institutos privados de investigación científica. In: CONFERENCIA EN EL INSTITUTO POPULAR DE CONFERENCIAS, 1945, Buenos Aires. Anais [...] Buenos Aires, 5 sept. 1945.

DE FANELLI, A.; MOGUILLANSKY. G. Profesión académica en la Argentina: carrera e incentivos a los docentes en las Universidades Nacionales. Revista de Política Educativa, Buenos Aires: UdeSA-Prometeo, año 1, n. 2, 2010.

DE SOUSA SANTOS, B. La universidad en el siglo XXI. Para una reforma democrática y emancipadora de la universidad. Umbrales: Revista del Postgrado en Ciencias del Desarrollo, La paz: Cides-umsa, Asdi y Plural editores, n. 15, p. 13-70, 2007. 
FEDERACIÓN NACIONAL DE DOCENTES UNIVERSITARIOS. EI SiDIUN: un nuevo capítulo de la reforma neoliberal y autoritaria para las universidades. [S. l.]: CONADU, 10 mayo 2019. Disponible en: https://conadu.org.ar/el-sidiun-un-nuevo-capitulo-de-la-reforma-neoliberal-y-autoritaria-para-las-universidades/. Acceso en: 9 abr. 2020.

FERNÁNDEZ, M. A; LEMOS, María Luisa; WIÑAR, David. La Argentina fragmentada: el caso de la educación. Buenos Aires: Miño y Dávila, 1997.

GENTILI, P. A universidade na penumbra: o círculo vicioso da precariedade e a privatização do espaço público. In: GENTILI, P. (org.). Universidades na penumbra: neoliberalismo e reestruturação universitária. São Paulo: Cortez, 2001. p. 97-128.

KROTSCH, P.; SUASNÁBAR C. Los estudios sobre la educación superior: una reflexión en torno a la existencia y posibilidades de construcción de un campo. Revista Pensamiento Universitario, v. 10, n. 10, p. 35-54, 2002.

LEAL, M. La vida profesional y tareas intelectuales de dos comunidades académicas de la UNT y sus patrones de respuestas frente al Programa de Incentivos. In: PONENCIA EN EL TERCER ENCUENTRO NACIONAL “LA UNIVERSIDAD COMO OBJETO DE INVESTIGACIÓN”, 2002, La Plata. Anais [...] 24 y 25 oct. 2002.

NAIDORF, J. La privatización del conocimiento público en universidades públicas. In: GENTILI, P.; LEVY, B. (comp.). Espacio público y privatización del conocimiento. Buenos Aires: CLACSO, 2005. p. 101-162.

NAIDORF, J. Los cambios en la cultura académica de la universidad pública. Buenos Aires: Eudeba, 2009.

NAIDORF, J.; PERROTTA, D. La educación superior en Argentina. Algo de ayer, un poco de hoy y pistas de mañana. In: TEODORO, A. (ed.). Revista A Educação superior no espaço lberoamericano. Do elitismo à transnacionalização. Lisboa: Edições Universitárias Lusófonas, 2010.

NAIDORF, J.; PERROTTA, D. La privatización del acceso abierto. Nuevas formas de colonización académica en América Latina y su impacto en la evaluación de la investigación. Revista Universidades, n, 73, p. 41-50, 2017.

RINESI, E. ¿̇Cuáles son las posibilidades reales de producir una interacción transformadora entre Universidad y Sociedad? Documentos para el debate, v. 201, 2012. Disponible en: http://cidac. filo. uba. ar/sites/cidac. filo. uba. ar/files/revistas/adjuntos/Clase. Acceso en: 9 abr. 2020. 
SARTHOU, N. Entendiendo la política pública a través de sus instrumentos: el programa de incentivos a docentes investigadores de universidades nacionales. Revista DAAPGE, Santa Fe, Argentina: UNL, n. 22, p. 71-102, 2014.

VOMMARO, P. Reforma y ajuste en la Universidad de Buenos Aires. Revista Memoria, México DF: Boletín del Centro de Estudios del Movimiento Obrero y Socialista, n. 148, jun. 2001.

Endereço para correspondência: Puan 480, C1420 CABA, Argentina; judithnaidorf@gmail.com Roteiro, Joaçaba, U. 45, p. 1-22, jan./dez. 2020 | e23034 |E-ISSN 2177-6059 
